\title{
Competencias digitales docentes en época de emergencia sanitaria: necesidades y oportunidades para estudiantes de educación secundaria en Lambayeque
}

\author{
Dr. Angel Johel Centurión Larrea \\ Universidad San Martín de Porres \\ https://orcid.org/0000-0002-7169-7680 \\ acenturionl@usmp.pe
}




\title{
Competencias digitales docentes en época de emergencia sanitaria: necesidades y oportunidades para estudiantes de educación secundaria en Lambayeque
}

\begin{abstract}
Resumen
El estudio analiza la percepción del desarrollo de las competencias digitales docentes (CDD) en el período de emergencia sanitaria por la covid-19 durante el año 2020, distinguiendo entre el efecto inicial, las estrategias de respuesta luego del primer momento y las tareas a tomar en cuenta para el año escolar 2021. El estudio incluyó la participación de 75 docentes de 12 instituciones educativas de Lambayeque; 3 especialistas de educación; y 6 docentes, 2 directivos y 6 estudiantes de nivel secundario. Los instrumentos que se aplicaron fueron una encuesta, entrevistas a profundidad y grupos focales. Entre los resultados más relevantes, destaca la presencia de experiencias muy innovadoras que siguieron a la frustración y la preocupación inicial en el nuevo escenario condicionado por el uso de la tecnología. Además, el desarrollo de las CDD varía según el tipo de institución, zona geográfica, involucramiento de los familiares, acceso a la tecnología y nivel de comunicación institucional que favorezca el trabajo colegiado. No obstante, se reconoce la labor docente para hacer frente a los factores condicionantes, sobre todo, los económicos, emocionales y sociales. En cuanto a la tecnología, se evidencian heterogeneidades en la brecha de acceso, dominio y uso efectivo de la misma. Se reconoce, así, la necesidad de trabajar mucho más en la retroalimentación efectiva a partir de un mejor control del accionar del estudiante y sus familias. En líneas generales, se demanda de un plan integral de formación docente en competencias digitales que contemple las distintas esferas que condicionan el proceso.

Palabras clave: Pandemia, educación a distancia, competencias del docente, educación básica, aprendizaje a distancia
\end{abstract}




\title{
Digital Teaching Skills in a Time of Health Emergency: Needs and Op- portunities for High School Students in Lambayeque
}

\begin{abstract}
This article analyzes the perception of the development of DTC digital teacher competencies in the period of sanitary emergency due to Covid-19 during the year 2020, distinguishing between the initial effect, the response strategies after the first moment, and the tasks to be considered for the 2021 school year. The study included the participation of 75 teachers from 12 educational institutions in Lambayeque; 3 education specialists; and 6 teachers, 2 managers and 6 secondary level students. This research applied instruments such as survey, in-depth interviews and focus groups. Results evidences the presence of very innovative experiences that have followed the frustration and initial concern in the new scenario conditioned by technology. The study also shows that the development of the DTC varies according to the type of institution, geographical area, involvement of family members, access to technology and level of institutional communication that favors collegial work. However, teaching work faced conditioning factors, especially economic, emotional, and social ones. Technological heterogeneities are expressed in the access gap, domain and effective mastery of them. Thus, the study shows the need to work on effective feedback from a better control of the actions of the student and their families. In general, current context demands for a comprehensive teacher training plan in digital skills that includes the different areas that condition the process.
\end{abstract}

Keywords: Pandemic, distance education, teacher skills, basic education, distance learning 


\section{Introducción}

La educación es un sistema considerado en todos los lugares del mundo como una vía para el desarrollo (Gehrig y Muñoz, 2015). Sin embargo, desde siempre, ha revelado brechas de distinta índole que testimonian diferencias entre grupos sociales y económicos. Una de ellas es la brecha tecnológica, la misma que demanda no solo un mayor acceso, sino también el desarrollo de competencias digitales de docentes y estudiantes para poder aprovecharla en beneficio del desarrollo de la sociedad misma.

En el año 2020, esta brecha tecnológica ha sido bastante visible debido a la emergencia sanitaria producida por la covid-19, epidemia que se dio en todo el planeta y que se ha constituido como una amenaza para el avance de la educación. Para ello, basta con evidenciar el cierre inicial de las instituciones educativas de todos los niveles; el despido de docentes o la disminución de sus remuneraciones; la pérdida de oportunidades de aprendizajes, al no desarrollarse lo proyectado en el currículo escolar (Dans, 2020; Ruiz, 2020; Viner et al., 2020); y la deserción y el incremento de la desigualdad en el acceso y uso de la tecnología para estudiar, que, si bien no es el único factor preocupante en torno a la tecnología, es un signo muy claro de la brecha digital creciente relacionada con la educación (Failache, Katzkowicz, y Machado, 2020). Esta brecha se ha manifestado de distintas formas en este contexto. Mientras algunos estudiantes pudieron seguir clases a distancia por tener conexión permanente en sus hogares, e incluso cuentan con más de un dispositivo tecnológico, y operadores telefónicos diferentes, otros deben salir de sus hogares y caminar largas distancias para tratar de conectarse a una red y tener acceso a las clases. Del mismo modo, mientras algunos pueden desaprovechar sus datos en asuntos sin importancia, otros no pueden enviar la tarea encomendada por falta de los mismos.

Posturas de destacados líderes mundiales - entre ellos, el Secretario General de la ONU - han visto el problema como una gran oportunidad para mejorar las condiciones en las que se desarrolla la educación, y para optimizar sus procesos y recursos, entre los que resalta una mejor infraestructura tecnológica. Se recomienda, entre otros, migrar a sistemas de calidad que aseguren el desarrollo sostenible de los pueblos, considerando la alfabetización y la infraestructura digitales que aseguren el desarrollo de los procesos educativos (Guterres, 2020).

En América Latina, para hacer frente a los efectos de la pandemia en el sector educación, se ha optado por las modalidades a distancia con o sin tecnología, apoyo y movilización de personal y comunidades educativas, y atención al bienestar integral de los estudiantes (Vargas et al., 2021). El personal docente y administrativo ha demando asegurar conexiones adecuadas y monitorear el aprendizaje de sus estudiantes de forma permanente, y con diversos y nuevos formatos e instrumentos (Cepal y Unicef, 2020). Incluso antes de la pandemia, ya se requería de formación en herramientas TIC para la enseñanza en 110 I la educación inicial. Además, un gran porcentaje de directivos afirmaban que 
sus instituciones tenían tecnología digital inadecuada o insuficiente (OCDE, 2019). En países como Chile, por ejemplo, solo un $9 \%$ de docentes consideraba que sus estudiantes contaban con hábitos para estudiar de manera autónoma y el 25\% afirmaba que sus alumnos ostentan pocas habilidades para usar aplicaciones de trabajo a distancia (Salazar, 2020).

El contexto actual evidencia varios desafíos. El primero está relacionado con la necesidad del docente - que se puede convertir en desesperación- de comunicarse virtualmente con sus estudiantes cuando otros usan al mismo tiempo la tecnología en su hogar, pues los dispositivos se recargan, la red no soporta a tantos conectados y él tiene la carga adicional de conducir el proceso educativo: verificar quiénes entran y quiénes responden a las distintas estrategias que ha trazado para la reunión virtual; retroalimentar permanentemente a cada estudiante; entre otros aspectos que, al no controlarlos, terminan por generar estrés (Moreno et al., 2021). En segundo lugar, se han roto las normativas de la privacidad y protección de datos, pues el docente ha tenido que ingeniárselas de diversas formas para llegar a sus estudiantes. Ello ha significado organización y confusión (Cabrera, 2020) muchas veces, porque, mientras que en la presencialidad dominaba estrategias didácticas, ahora en la virtualidad ve que no puede incorporarlas con el mismo efecto (Krumsvik, 2011). Por ejemplo, estudiantes de zonas rurales alejadas interactúan con sus docentes por mensajería personal o por redes sociales, en la mayoría de casos, solo de manera asincrónica. Este contexto ha demandado la comunicación permanente por parte del docente con los padres de familia para explicarles nuevas formas de interacción, distintas a las prestablecidas, que implica el apoyo por parte de ellos para lograr los objetivos académicos propuestos.

En este escenario, las competencias digitales de los docentes (CDD) se han vuelto indispensables. Estas son entendidas tanto como instrumentos, como resultados y procesos empoderadores. Se constituyen como instrumentos, en tanto facilitan el desarrollo de la innovación en los estudiantes y exigen del docente combinar actitudes, conocimientos y procesos (Marza y Cruz, 2018). Asimismo, son resultados, en la medida que están ligadas a procesos de alfabetización digital que deben de ser prácticos y medibles en función de sus resultados en el desarrollo efectivo de aprendizajes (Iordache et al., 2017). Las CDD también son procesos empoderadores fundamentales para el desarrollo de la ciudadanía plena en la interacción con los diversos ámbitos de la sociedad (Marzal y Cruz, 2018; Revelo, Revuela y Gonzáles, 2020).

Las competencias suponen un saber hacer en un contexto de manera pertinente, eficiente, crítica o autónoma (Ferrari, 2013), pero, en el caso de los docentes, esto ocurre en relación con lo educativo (Zahonero y Martín Bris, 2012 citado en Esteve, 2015). El Institute for Prospective Technological Studies (IPTS), luego de la consulta a varios expertos y modelos internacionales, cuenta con una propuesta que integra $21 \mathrm{CDD}$ agrupadas en cinco áreas o dimensiones (INTEF, 2017): 
(a) Información, que se refiere a identificar, localizar, recuperar, almacenar, organizar y analizar la información digital

(b) Comunicación, que implica comunicar en entornos digitales, compartir recursos, conectar y colaborar, e interactuar y participar en redes

(c) Creación de contenidos, en la que se trata de crear y editar contenidos multimedia nuevos, integrar y reelaborar, programar y aplicar los derechos de propiedad intelectual

(d) Seguridad, que implica la protección personal, de datos, de identidad digital, uso seguro y sostenible

(e) Resolución de problemas, que implica identificar y tomar decisiones para resolver disyuntivas teóricas y técnicas (Ferrari, 2013).

Estas definiciones reafirman la necesidad de que los docentes desarrollen un conjunto de CDD y abracen el paradigma que pone al estudiante como protagonista del proceso de enseñanza (Cervera, Martínez y Mon, 2016). No es suficiente que los educadores adquieran por cuenta propia sus competencias digitales (Esteve, 2015), sino que este proceso sea acompañado y mediado para el desarrollo efectivo de estrategias acordes a la virtualidad, que además deberán de responder a las necesidades específicas de cada realidad sociocultural en donde se lleva a cabo el aprendizaje (Pimienta, 2007). Esto es muy importante, porque supera el mero dominio de las redes sociales.

En Perú, los docentes se enfrentaron a condicionantes parecidas al resto de Latinoamérica. Las respuestas no se hicieron esperar y se sucedieron un conjunto de acciones desde las políticas de Estado para hacer frente a la situación. El trabajo de docentes y directivos fue clave para asegurar la continuidad de la tarea. Esto implicó, para la mayoría, una nueva práctica y una preocupación constante por mejorar acciones y procesos. Así, en abril del 2020, se dio en el país el inicio de la enseñanza bajo la modalidad a distancia mediante la estrategia Aprendo en Casa, aplicada en la Educación Básica Regular (EBR). Esto constituyó un reto para los docentes peruanos, dado que, en el país, solo el $40,1 \%$ de los hogares cuenta con internet en la zona urbana y solo el $6 \%$ en la rural (INEI, 2020, citado en IPE, 2020), aunque la utilización del celular supera el $80 \%$ en promedio a nivel nacional. Una encuesta nacional aplicada a padres de familia, realizada por el Instituto Peruano de Economía (IPE) en el 2020, concluyó como dificultades más relevantes la falta de materiales y de tiempo para el acompañamiento a las actividades de sus hijos: el 46,1\% de los padres siente que necesita más ayuda para guiarlos. Jaime Saavedra, director global de Educación del Banco Mundial, señala que uno de los desafíos del país implica cerrar la brecha digital dentro y fuera del hogar a una mayor velocidad (IPE, 2020). En diversas oportunidades, el Ministerio de Educación (Minedu) ha destacado la importancia del rol del docente, principalmente, en el acompañamiento a sus alumnos en este escenario adverso, en tanto son los maestros quienes deben brindar propuestas situadas a cada realidad específica 112 I marcada por carencias, limitaciones, distorsiones, y empirismos normativos 
y aplicativos en todo nivel, más aún en un escenario marcado por el uso de la tecnología.

Se reconoce que la adecuación a este nuevo escenario no debió ser tan abrupta, puesto que el desarrollo de las TIC exigía desde antes de la pandemia el desarrollo de competencias digitales tanto en docentes como estudiantes (Hernández et al., 2014; Hinostroza, 2017). Un gran porcentaje de currículos de formación básica en América solo contemplan el desarrollo de habilidades en torno a la computación (Banco Mundial, 2018). Desde hace más de una década, el Diseño Curricular Nacional (DCN) se orienta hacia las competencias básicas; asimismo, el actual Currículo Nacional del Perú incorpora la competencia transversal 28: se desenvuelve en los entornos virtuales generados por las TIC (Minedu, 2016). Incluso, el Ministerio de Educación, a través del Marco de Buen Desempeño Docente, promueve el fortalecimiento del desempeño 23: Utiliza recursos y tecnologías diversas y accesibles, y el tiempo requerido en función del propósito de la sesión de aprendizaje (Ministerio de Educación, 2020). Esto no solo evidencia la existencia de un marco para facilitar el desarrollo de la competencia digital de los sujetos educativos, sino que expresa la incompetencia de las instituciones formativas para fortalecerla, y la ineficacia de los órganos directivos para acompañarla y desarrollarla.

En Lambayeque, región ubicada al norte de Perú, los docentes de EBR han tenido que enfrentar también este escenario al que no estaban preparados y en el cual sus competencias digitales se han puesto de manifiesto con todas sus carencias y potencialidades, frente a lo cual progresivamente han asumido acciones de mejora (Luna y Larrea, 2020). En una consulta nacional sobre propuestas regionales para el Pacto Perú ${ }^{1}$ presentada por la Mesa de Concertación para la Lucha contra la Pobreza en Lambayeque (MCLCP, 2020), se estableció como uno de los problemas la limitación al acceso a la educación virtual por la débil conectividad que afecta a docentes y estudiantes; el limitado recurso humano para el monitoreo y la orientación de los sujetos involucrados en el proceso como docentes, estudiantes y familias; $y$ las brechas de conocimiento de maestros a quienes les cuesta ingresar al mundo digital. Para esta última, se plantea una alfabetización digital que, según la consulta, debe ser asumida por las unidades de gestión educativas locales (UGEL) y la Gerencia Regional de Educación (GRED) (Mesa de Concertación para la Lucha contra la Pobreza, 2020).

El escenario en el futuro inmediato parece ser que no va a cambiar demasiado. En ese sentido, se necesita caracterizar la experiencia docente en torno al desarrollo de su competencia digital en este período: identificar causas, consecuencias y alternativas relacionadas a la misma, las que servirán de

1. En atención a la demanda ciudadana de unidad, el presidente Martín Vizcarra convocó al Pacto Perú para que todas las fuerzas políticas construyan un nuevo acuerdo básico que ayude a los peruanos a enrumbarse al proceso de transición política, con acuerdos mínimos que den marco al debate necesario que se desarrollará en la próxima campaña electoral. 
mucho para lograr mejores aprendizajes en los estudiantes, fin último de la educación en el Perú (Minedu, 2019).

Esta investigación analiza la percepción del desarrollo de las competencias digitales docentes (PCDD) desde los condicionamientos producidos por la pandemia sanitaria de la covid-19 durante el año 2020 en docentes de secundaria, sus estudiantes, directivos y especialistas de la región Lambayeque. Se entiende por percepción a la formación de impresiones, que establecen factores subjetivos a las personas con respecto a sí mismas, el objeto percibido y el contenido que se trata (Paéz y Márquez, 1999, citados en Arias, 2006). En este caso, las percepciones se hacen en alusión a las dimensiones de información y alfabetización informacional, comunicación y colaboración, creación de contenido digital, seguridad y resolución de problemas, en torno a tres momentos: inicio, desarrollo y expectativas de actuación inmediatas futuras.

Conviene preguntarnos, entonces, ¿cómo se perciben las características de la CDD por parte de los docentes de secundaria y sus estudiantes? ¿Qué factores han resultados propicios y cuáles no? ¿Qué podría servir para replicar en otras instituciones educativas? ¿Cuáles son las tareas pendientes en torno a las CDD? Para ello, la investigación se propuso como objetivo general analizar el desarrollo de las competencias digitales docentes (CDD) en el período de emergencia sanitaria por covid-19 durante el año 2020, distinguiendo como objetivos específicos analizar el efecto inicial, analizar las estrategias de respuesta luego del primer momento y proponer tareas a tomar en cuenta para el año escolar 2021.

\section{Metodología}

\section{Participantes}

La investigación contó con la participación de docentes, directivos y estudiantes. En primer lugar, formaron parte del estudio 75 docentes, seleccionados a partir de dos criterios de inclusión: que hayan desarrollado clases durante todo el 2020 y que realizaran la encuesta publicada en el portal de las Unidades de Gestión Local (UGEL) de las tres provincias de la región. También, participaron tres especialistas en temas de educación que pertenecían a cada una de las provincias de la región Lambayeque, además de catorce participantes en dos grupos focales (ocho docentes y directivos, y seis estudiantes) (ver Apéndice).

Con respecto a los docentes, el propósito fue cubrir la heterogeneidad de la región estudiada, pero respetando la representatividad de los diversos grupos que lo conforman. Por este motivo, se tomó en cuenta el régimen educativo (público o privado), la zona (urbana o rural) y el área geográfica (tres provincias). Así, se seleccionaron en total doce instituciones: seis de Chiclayo, tres de Ferreñafe y tres de Lambayeque. Los tres especialistas y catorce participantes restantes completaron la observación base para el análisis e interpretación para conocer a profundidad los factores condicionantes del desarrollo de las CDD. 


\section{Instrumentos de recolección de información}

El instrumento eje fue la encuesta sobre CDD. Para ello, se adaptó el cuestionario Matriz de Competencias Digitales del Profesorado para la Sociedad del Conocimiento, propuesto por Katya Pozo Pérez y José Tejada Fernández (2018), validado para el estudio por dos expertos en tecnologías de la información y la comunicación. En este, se contemplan dos partes. En la primera, se observa la dimensión "Actuación" con preguntas cerradas con escala Likert (siempre, casi siempre, ocasionalmente, casi nunca, nunca), que observaban las competencias digitales: planificación de los aprendizajes mediante las TIC, desarrollo de los aprendizajes a través de las TIC, evaluación formativa mediante TIC, desarrollo profesional, y compromiso ético en las TIC. En la segunda, se indaga con preguntas abiertas sobre las dimensiones: condicionantes, lecciones aprendidas y proyecciones. El estudio se complementó con grupos focales y entrevistas a profundidad. Para dicho recojo de información, se precisaron sendas guías semiestructuradas, para las cuales se determinaron categorías, subcategorías y unidades de análisis. Todos los instrumentos fueron validados por tres expertos en educación.

La aplicación fue virtual en los tres casos, luego de coordinaciones con directores, el decano del Colegio de Profesores de Lambayeque, docentes y estudiantes. Asimismo, se siguieron los protocolos éticos del consentimiento informado.

\section{Procedimiento}

Esta investigación se ha desarrollado bajo el enfoque mixto y en un nivel exploratorio-descriptivo. Se considera exploratorio, porque el problema a investigar ha sido poco estudiado en el contexto y se busca la familiarización de un fenómeno relativamente desconocido (Hernández, Fernández y Baptista, 2014), en este caso, los condicionamientos propios de la pandemia por covid-19, fenómeno que fue intempestivo. Además, es descriptivo, en tanto busca, en palabras de Danhke (1989), "especificar las propiedades, las características y los perfiles importantes de personas, grupos, comunidades o cualquier otro fenómeno que se someta a un análisis" (citado en Hernández, Fernández y Baptista, 2014, p.117). Dentro de los diseños mixtos, se ha seleccionado la triangulación concurrente (Ditriac), que "se utiliza cuando el investigador pretende confirmar o corroborar resultados y efectuar validación cruzada entre datos cuantitativos y cualitativos, así como aprovechar las ventajas de cada método y minimizar sus debilidades" (Hernández, Fernández y Baptista, 2014, p. 557). Después de la codificación de los datos recogidos durante la investigación, se fue procesando la información utilizando la estadística. Finalmente, se realizó el proceso de triangulación final para obtener los resultados definitivos. 


\section{Resultados}

Al inicio del año 2020, luego de que el presidente de la República, Martín Vizcarra Cornejo, comunicara el inicio de las clases a distancia el 6 de abril, los docentes lambayecanos se sintieron, en general, retados, pero también experimentaron frustraciones, desesperación y estrés. La gran mayoría de ellos asumía que no dominaban las competencias básicas que exige la docencia a distancia.

Un primer aspecto a destacar, en relación con las percepciones respecto a la repuesta inicial que produjo la pandemia en las CDD, es que el bajo porcentaje de docentes $-10 \%$ aproximadamente- expresa que ya habían recibido capacitaciones en entornos virtuales de aprendizaje antes de dicha crisis, y coinciden que estas no desarrollaban las habilidades necesarias que contemplan las dimensiones de planificación, desarrollo y evaluación mediadas por la tecnología. La situación se agravó por la poca oferta de capacitaciones en el tema, que se sumó al escenario económico, social, y emocional de ellos y sus estudiantes, producido por la pérdida de trabajo de familiares, la enfermedad de los mismos y la disrupción del uso de la tecnología. En las zonas rurales de la región y en las instituciones de régimen privado, esto es más grave aún, según se concluye a partir de las entrevistas y grupos focales (E2, E3, Ia5, Ia8).

El trabajo colegiado, entendido como trabajo en equipo docente para hacer frente a las necesidades educativas, ha resultado muy acertado en muchas instituciones que han empezado a desarrollar acciones disciplinares e interdisciplinares para el logro de sus objetivos profesionales. Sin embargo, ello ha resultado aún difícil en instituciones con gran cantidad de docentes. Los entrevistados coinciden, asimismo, que la presencia del Estado ha sido insuficiente, con lineamientos que no han sido los mejores en función de la realidad de la región, por lo que el liderazgo pedagógico de los directivos ha sido un factor preponderante, aunque a veces contradictorio.

Por un lado, mi director me llamaba para pedirme que no me estrese, pero antes de terminar la llamada me recordaba las tareitas: el informe tal, la reunión tal, las llamadas y coordinaciones tales. Esto, sumado a nuestras reuniones por la mañana y clases por la tarde, y las llamadas de los padres a toda hora, terminaron por afectar a mí y a mi familia. (Ia7)

En suma, el primer momento del desarrollo de las competencias digitales docentes en la región Lambayeque durante el 2020 se percibe como caótico, desordenado y acéfalo en la mayoría de los casos, pero, desde el inicio de la emergencia sanitaria, el docente ha asumido también el reto por cambiar dicha situación. Por ejemplo, uno de ellos indicó: "Es sorprendente cómo, pese a la falta de claridad, el docente ha sido comprometido con su misión social. En su gran mayoría, ha tenido que innovar su propia práctica para llegar de la mejor manera a sus estudiantes" (E1). 
Los profesores sí han estado siempre llamándonos, preguntaban por cómo nos iba, pese a que en algunos casos ellos también tenían familiares enfermos, recuerdo que a pesar de que no sabían usar Zoom al inicio poco a poco fueron mejorando. (Ib4)

Un segundo aspecto a destacar es la percepción sobre la planificación, el desarrollo y la evaluación formativa de los aprendizajes, así como acciones en torno al desarrollo profesional y al compromiso ético de los mismos. Con respecto a la planificación, el $30 \%$ de los docentes encuestados asume que nunca o casi nunca diseña y desarrolla materiales de aprendizaje innovadores con TIC, y solo el 23,5\% explora críticamente nuevos sistemas tecnológicos y redes de colaboración y gestión del conocimiento para contribuir a la potenciación del aprendizaje y creatividad en los alumnos en la generación del conocimiento, como se aprecian en las Fig. 1 y Fig. 2 respectivamente.

Figura 1

Desarrollo de materiales de aprendizaje innovadores con TIC
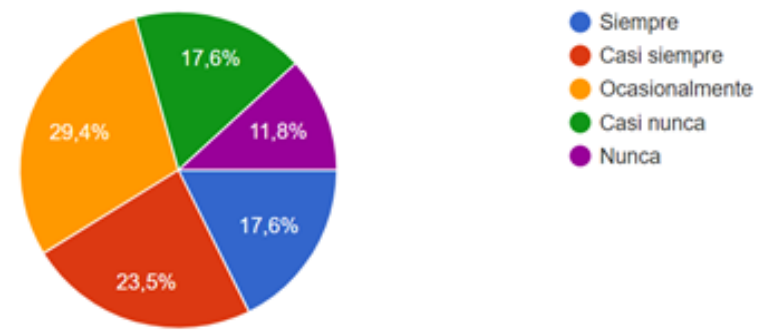

Fuente: Encuesta de PCDD aplicada a docentes de la región Lambayeque

Figura 2

Exploración crítica de nuevos sistemas tecnológicos y redes de colaboración y gestión del conocimiento

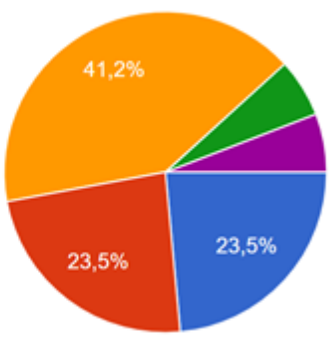

Siempre

Casi siempre

Ocasionalmente

Casi nunca

Nunca

Fuente: Encuesta de PCDD aplicada a docentes de la región Lambayeque. 
En cuanto al desarrollo de los aprendizajes de los estudiantes en entornos mediados por la TIC, se puede afirmar que la incipiente capacidad de dominio de la tecnología para la enseñanza se agravó por las limitaciones de los docentes de educación secundaria lambayecanos y sus estudiantes debido al acceso a la misma. A ello se sumaron problemas permanentes de conectividad; problemas emocionales por el contagio de la enfermedad; y sociales de diversa índole, como el incremento de la violencia, el desempleo, saboteos a los encuentros virtuales por el mal uso de las mismas (Ia1, Ia2, Ia6, Ib1, Ib4, Ib6).

Además de clases por radio y televisión, se contempló el uso del WhatsApp, de gran aceptación por la mayoría. Entre otras experiencias, se encuentra el uso de plataformas virtuales - como Edmodo, Moodle, sianet- y herramientas para videoconferencias - como Meet, Zoom, Skype, entre otras. A partir de las entrevistas, se concluye que solo el $17,6 \%$ de los docentes asume que siempre gestionó ambientes y plataformas de aprendizaje en red para desarrollar nuevas experiencias colaborativas de aprendizaje que permitan a los alumnos resolver situaciones complejas, ya sea simuladas o de la realidad (Fig. 3). Según los mismos docentes, algunos de sus colegas se limitaron a reproducir las guías proporcionadas por la estrategia estatal Aprendo en Casa, sin mayor adecuación, ni seguimiento (E1, E2, E3, Ia2, Ia4).

Figura 3

Gestión de ambientes y plataformas de aprendizaje en red para desarrollar nuevas experiencias colaborativas de aprendizaje
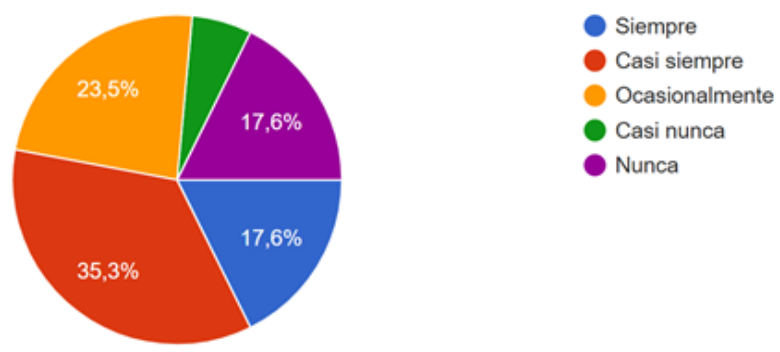

Fuente: Encuesta de PCDD aplicada a docentes de la Región Lambayeque.

La evaluación formativa de los aprendizajes es, a partir de lo mencionado por los estudiantes, el principal reto de los docentes lambayecanos. Sobre este tema, la mayoría de entrevistados señalaron que necesitan una mayor exigencia y un acompañamiento efectivo.

Los docentes hasta el final del año se han contentado con que solo le presentemos las actividades y sus preguntas, en su mayoría, han sido sobre si las cumplimos o no, pero la mayoría no nos ha dicho si estaba bien. Debe de haber otras estrategias, porque solo he sido una máquina 
de hacer tarea tras tarea-hablo de la mayoría. Y, además, no es justo que algunos compañeros que se aparecieron al final les pongan la misma nota. Algunos sí entraban a las reuniones que organizábamos para otras cosas, pero a las clases no. Eso debe de mejorar. ¿Verdad, chicos? (Ib5)

Esto se reafirma con las respuestas de la encuesta, en la que los mismos docentes reflexionan respecto de su accionar en relación con la evaluación formativa. Como se aprecia en la Fig. 4, el 52,9 \% menciona que solo ocasionalmente provee a sus alumnos el acceso a múltiples perspectivas y contenido. Es decir, tienen limitaciones para virtualizar contenidos de aprendizaje, así como variadas formas de evaluación y retroalimentación mediadas por las tecnologías de la información y la comunicación.

Figura 4

Suministro de acceso a múltiples perspectivas y contenidos, así como variadas formas de evaluación y retroalimentación mediadas por TIC
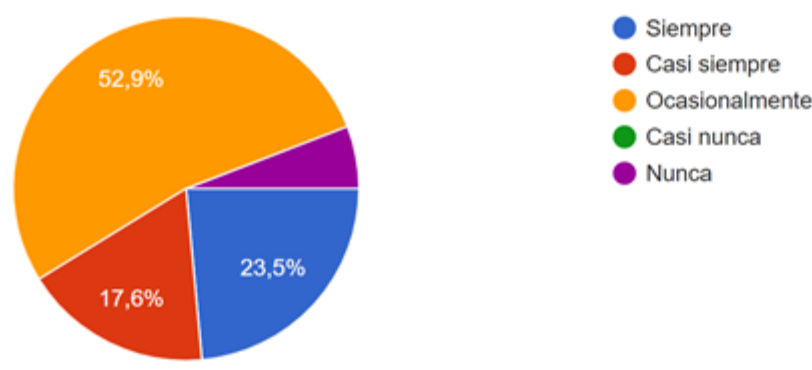

Fuente: Encuesta de PCDD aplicada a docentes de la Región Lambayeque.

Con respecto al desarrollo de la profesionalidad docente, que involucra las tecnologías de la información y la comunicación, se puede afirmar que, a decir de los directivos, estudiantes y de los propios docentes consultados en las entrevistas y grupos focales (E1, E3, Ia2, Ia6, Ia8, Ib2, Ib5, Ib6), el período de emergencia sanitaria por covid-19 ha sido beneficioso para el avance de las competencias digitales. Sin embargo, conviene aprovechar este contexto para que, desde el Estado, de manera organizada y sensibilizándose con este condicionamiento, se configuren capacitaciones, iniciativas de las mismas instituciones y de los mismos docentes, para lograr su desarrollo significativo a mediano y largo plazo. Los entrevistados reconocen que la virtualidad ha venido para quedarse y que es una necesidad capacitarse, pues se prevé que cada vez la educación la incorporará más y más. 
Figura 5

Creación de redes virtuales para estimular la innovación y la calidad mediante la reflexión y el intercambio mutuo

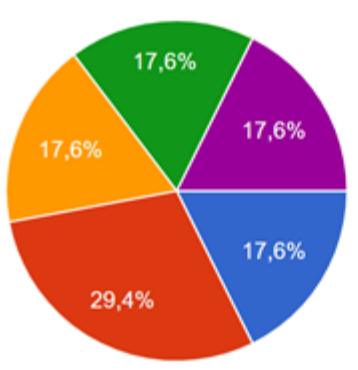

Siempre

Casi siempre

Ocasionalmente

Casi nunca

Nunca

Fuente: Encuesta de PCDD aplicada a docentes de la región Lambayeque.

Como se puede apreciar en la Figura 5, el 17,6\% de los encuestados afirma que siempre crea redes virtuales para estimular la innovación y la calidad mediante la reflexión docente. El mismo porcentaje señala que nunca lo hacen, casi nunca y ocasionalmente, mientras que el $29,4 \%$ afirma que lo hace casi siempre.

En cuanto al compromiso ético, un grupo considerable afirma que actúa con criterios éticos para la integración responsable de las TIC en el currículo y en las actividades de aprendizaje de los alumnos (Fig. 6). El 35,3\% indica que siempre lo hace; el $41,2 \%$, que casi siempre; el $17,6 \%$, que ocasionalmente; y solo el 5,6\% señala que casi nunca lo hace. No obstante, los especialistas entrevistados plantean que es necesario trabajar en ello como requisito para que luego el mismo docente pueda exigir a sus estudiantes.

Muchos estudiantes plagian los trabajos de sus propios compañeros, se las hacen sus familiares o simplemente las bajan de Internet. Por ello, el comportamiento ético de los colegas docentes de la región debe de involucrar un modelamiento desde sus propias prácticas en las que no solo realicen el seguimiento efectivo de los productos de sus estudiantes, sino que también propongan y lideren innovaciones. Superando una visión disciplinar de las competencias por otra que contempla el trabajo interdisciplinario. (Ic2) 
Figura 6

Actuación con criterios éticos para la integración responsable de las TIC en el currículo y en las actividades de aprendizaje de los alumnos
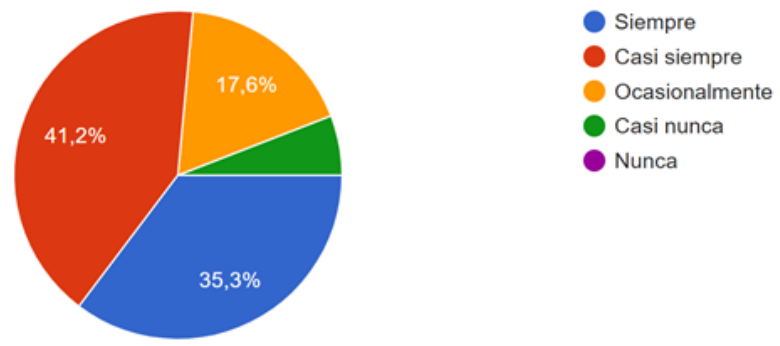

Fuente: Encuesta de PCDD aplicada a docentes de la región Lambayeque.

Sobre las relaciones interpersonales entre docentes, docentes y directivos, docentes y padres de familia, y docentes y sus estudiantes, los entrevistados afirman que la situación extrema experimentada les permitió conocer más a sus estudiantes. Es decir, se convirtió en una oportunidad, fruto del seguimiento de los aprendizajes. En algunos casos, la situación fue aún mejor que en la presencialidad, pues conversaron con las familias; conocieron el contexto y las necesidades económicas, sociales y tecnológicas de las mismas; se identificaron con ellas. Asimismo, el acercamiento con sus colegas también ha sido muy beneficioso.

En mi caso particular, he tenido que reencontrarme como persona, vivir mi vocación, dar más tiempo que en la presencialidad, pues deberíamos actuar en un escenario extraño hace unos meses y que demandaba hacer atractiva la clase para que los estudiantes efectivamente logren sus aprendizajes. Esto es un reto constante que no debemos de continuar. Para ello, es importante conocer a nuestro estudiante y empatizar con su realidad, pues muchos de ellos efectivamente no tenían los medios mínimos para sus clases y lo peor de todo que parece que vamos a seguir así todavía. A mí también, a veces, me ha fallado el Internet, así que me acostumbré a ser flexible. También, quiero decir que me ha ayudado mucho las reuniones con los colegas: el escucharnos y compartir nuestras experiencias, necesidades, y trabajar colaborativamente ha sido muy importante. (Ia4)

Asimismo, a partir de las entrevistas, resalta la relevancia de la relación escuela-familia, pues se ha experimentado — más que en otros momentos - la importancia de los padres y demás familiares en la formación de los adolescentes. En algunos casos, estos han apañado malos comportamientos y, en otros, su exigencia ha sido determinante para lograr mejoras de aprendizajes. Se ha sentido, también, el efecto de los hogares disfuncionales. 
En lo que concierne a la tecnología, se ha superado el paradigma de una enseñanza aislada $-\mathrm{y}$ hasta sancionada - de las TIC, en que los celulares, laptops u otros dispositivos tecnológicos eran prohibidos, y su uso educativo era percibido como muy lejano en la mayoría de instituciones educativas, según se desprende de las entrevistas y grupos focales (E1, E2, E3, Ia2, Ia3, Ia5, Ia7). Se ha identificado que docentes y estudiantes usaban las redes sociales, pero solo con fines de interacción social; sin embargo, cobra otra dimensión en lo educativo y, por tanto, demanda formar al estudiante para su uso y que este sea efectivo con esos fines. Los docentes en su gran mayoría afirman que han aprendido nuevas acciones, herramientas, plataformas de aprendizaje; sin embargo, asumen en su gran mayoría, no estar conformes con el nivel actual de dominio, por lo que demandan ser capacitados.

En relación con el logro efectivo de aprendizajes, un número significativo de docentes afirma que es necesario y sumamente provechoso el trabajo colaborativo entre sus pares, así como las reuniones periódicas entre estos y los directivos de la IE. Los entrevistados agregan que han comprendido mejor que es muy importante que se atienda al estudiante en todas las esferas que condicionan su aprendizaje para, a partir de ello, planificar las acciones pedagógicas futuras. Se sienten impotentes en algunos casos por no poder ayudar más allá de sus posibilidades las carencias de sus estudiantes; no obstante, reafirman su compromiso por la mejora continua.

Finalmente, se asume que las proyecciones del desarrollo de las competencias digitales de los docentes de educación secundaria deben de ejecutarse en lo personal, lo institucional y lo social. Con respecto a sus experiencias personales, los sujetos involucrados en el estudio perciben en general que los docentes deben capacitarse para lograr un mejor desarrollo y acompañamiento de los aprendizajes de los estudiantes; así, podrían transitar de consumidores a productores de productos tecnológicos. En la esfera institucional, urge diseñar o implementar plataformas digitales para el desarrollo más efectivo de las clases, fortalecer el trabajo colegiado, y proyectar acciones para el involucramiento de las familias y la salud emocional de docentes, estudiantes y otras personas implicadas en el proceso de aprendizaje. En el ámbito social, es necesario acortar la brecha digital, así como trabajar la gestión de las emociones a través de acciones proyectadas desde las instancias descentralizadas, como la Dirección Regional de Educación de Lambayeque; las Unidades de Gestión Educativa Locales; y, en general, desde el rol de Estado. La gran mayoría los participantes llegan al consenso de que sus acciones no han sido sentidas por dichas instituciones, por lo que reclaman políticas que sirvan realmente para facilitar su trabajo. 


\section{Discusión}

A partir de la literatura revisada y de los resultados obtenidos podemos establecer, respecto al primer objetivo específico, que la evaluación de la percepción del estado inicial de la competencia digital docente durante el período de emergencia evidencia que los docentes no se sienten preparados, que se vieron confundidos, frustrados y retados por la situación en general. Esto reafirma lo encontrado por Gerardo Picón, Gricelda Gonzales De Caballero y Juana Paredes (2020), quienes detectaron que, en el contexto de Paraguay, los docentes expresaron tener confianza, y, a la vez, estrés, ansiedad, sorpresa y - en menor porcentaje- miedo. En este marco, las clases bajo una modalidad distancia virtual eran asumidas como un compromiso profesional ineludible. La mayoría de los docentes opinaron que las dificultades para el desarrollo de las clases no presenciales se vinculaban con el colapso de la plataforma y la conexión de Internet.

Nuestro segundo objetivo específico correspondía al análisis de las estrategias docentes luego del primer impacto de la pandemia. Los resultados muestran que es necesario el desarrollo progresivo de la competencia digital docente, que supone que este transforme su rol, puesto que no es lo mismo actuar en la presencialidad y en lo virtual. Estos hallazgos se relacionan con lo manifestado por Laura Alonso y Florentino Blázquez (2016), quienes configuran como funciones del educador en la modalidad virtual las propias a su ejercicio docente, como diseñador de materiales didácticos, la de orientador, facilitador basado en una comunicación empática y comprensiva de sus estudiantes, y la función técnica a partir de conocimiento de las TIC. En Lambayeque, los docentes deben mejorar su actuación, tanto en la planificación de los aprendizajes como en el desarrollo de los mismos, en el monitoreo a partir de la evaluación formativa, en el desarrollo de su profesionalidad y en su compromiso ético en torno a las TIC, según lo mencionado por ellos mismos, sus estudiantes y especialistas. Esto es coherente con lo que concluyen Pozo y Tejada (2018), quienes consideran que "la competencia digital entraña un proceso de aprendizaje complejo, gradual y recurrente, que comprende la capacidad de utilizar adecuadamente las herramientas y recursos digitales para buscar, gestionar, analizar y transformar la información en conocimiento de manera crítica" (p. 77).

Los resultados ponen de manifiesto la necesidad de orientar a los alumnos, que deben ser capacitados para el uso de las tecnologías. Evidencian también la importancia de tener un nuevo rol de aprendiz, que asuma una mejora en la capacidad de organización y de la autodisciplina, debido a la libertad de la que dispone el estudiantado (Rugeles, Mora y Metaute, 2015). Asimismo, el contexto aumenta la necesidad de un estudiante capaz de lidiar con la incertidumbre y ambigüedad que supone el poseer diversas fuentes de conocimiento (Bautista, Borges y Forés, 2006). 
La educación virtual contribuye con la educación de carácter inclusivo, que implica la democratización de la educación (Thomas y Loxley, 2007) y sigue el modelo TPACK, que expresa que solo serán competentes los docentes que puedan activar de modo efectivo un conocimiento de triple naturaleza: disciplinar, pedagógico y tecnológico (Gisbert, González y Esteve, 2016). La investigación también revela la importancia de establecer evaluaciones de CDD como requisito para ejercer la docencia o como parte de la carrera profesional para, por ejemplo, lograr ascensos.

Por último, con respecto a las acciones inmediatas, se concluye que la capacitación es la tarea principal. Se recalca que esta debe incluir el desarrollo de habilidades para realizar el seguimiento de los aprendizajes, el trabajo colaborativo, el desarrollo de proyectos interdisciplinarios, la configuración de plataformas para cada institución, entre otras. Si bien es cierto que se contempla la autocapacitación y la capacitación institucional, se demanda un liderazgo más trascendente del Estado. Esto es reforzado por el estudio de Regina Alves, Teresa Lopes y José Precioso (2020) sobre el caso de Portugal, en que concluyen con evidencia exhaustiva que existe la necesidad de una mayor y más fuerte inversión en políticas públicas para la reforma y, en consecuencia, para la renovación de la clase docente. Coincide, además, con el estudio de Picón, De Caballero y Paredes (2020) en Paraguay, que plantea que es fundamental facilitar un trabajo en equipo y de acompañamiento en el aprendizaje profesional de los docentes, específicamente referido a sus competencias en el uso de las TIC para producir un aprendizaje más efectivo. Del mismo modo, es prioritario proporcionar a los profesores acceso a recursos en línea para el desarrollo y entrenamiento de herramientas de comunicación sincrónica, y lograr que avancen en estrategias basadas en el aprendizaje colaborativo en línea.

Según los resultados del presente trabajo, se demanda actuar frente a las exigencias de la sociedad del conocimiento partiendo de una alfabetización digital efectiva que permita no solo aprender a usar la tecnología, sino usarla de manera efectiva (Revelo, Revuela y Gonzáles, 2020), condicionada por factores de índole social y económico (Pimienta, 2007). En el actual estado de pandemia, urge atenderla sistémicamente, es decir, atender antes necesidades de salud, alimentación, empleo, entre otras, como condición necesaria. El docente ha asumido con singular compromiso su deber ante este difícil escenario, y, al parecer, las condiciones ideales no serán cubiertas en mucho tiempo, por lo que debe de reflexionar sobre lo actuado y asumir que, además del conocimiento adquirido hasta hoy sobre tecnología, deber caminar aún mucho más firmemente hasta integrarla en lo pedagógico y didáctico (Krumsvik, 2011), no dejando de lado las necesidades e implicancias que conlleva la formación digital de sus alumnos (Revelo, Revuela y Gonzáles, 2020). Es necesario, por tanto, seguir profundizando sobre este objeto de estudio atendiendo a algunas limitaciones propias del tamaño de la muestra, la observación virtual y el tipo de estudio. 


\section{Reflexión final}

En el 2021, en un contexto de celebraciones por el bicentenario de la proclamación de la independencia del Perú, las desigualdades se mantienen como una tarea pendiente. Aunque muchos han visto en la educación la mejor manera de lograr la equidad, parece todavía un lujo terminar la secundaria con docentes capacitados en competencias tan imprescindibles como la digital. Asimismo, es preciso priorizar la vacunación de maestros este año para retomar las clases presenciales (aunque algunas escuelas ya lo hicieron), así como dar un mayor acompañamiento a los docentes en las CDD para optimizar el uso de las Tablets, y otras herramientas, para el trabajo a distancia. Es en este contexto adverso, que los docentes peruanos deben asumir, una vez más, el gran reto de lograr aprendizajes en todas y todos los estudiantes del país.

En Lambayeque, segunda región más afectada por la pandemia en gran parte del 2020, las demandas de desarrollo de la competencia digital docente fueron respondidas en primer término por los mismos docentes; no obstante, requieren de un mayor apoyo de sus autoridades a todo nivel. Se deben asumir nuevos retos, aunque de manera diferenciada dada la heterogeneidad que expresa la región, según se trate de área; régimen; accesibilidad; y, en la actualidad, según el aprovechamiento de aprendizajes de la experiencia del año 2020. Si bien Lambayeque es una zona heterogénea, en Perú, hay regiones mucho más complejas por la misma articulación de territorio, lengua, niveles de pobreza, etc., por lo que se plantea el desafío de generar nuevos conocimientos a partir de esta investigación.

Los estudiantes esperan por su derecho a recibir una educación de calidad; por su parte, los padres de familia y demás familiares que los acompañan pueden colaborar aún más y mejor con el éxito de los objetivos de aprendizaje. Es imprescindible trabajar en sus competencias digitales y en el desarrollo de su autonomía plena, los mismos que se presentan hoy muy débiles. Sin duda, es una tarea que se deberá asumir a partir de un proyecto integral que involucre a toda la comunidad en pleno.

\section{Agradecimientos}

Se agradece a Dr. Jaime Montes García, Dr. Luis Alarcón Llontop, Mg. María del Perpetuo Socorro Cuzquén Cabrera, Mg. Víctor Huertas Esteves, Mg. Yen Marvin Bravo Larrea, Lic. Karim Castro Milian, Mg. Jesús Casas Montenegro, Lic. Socorro Granda Santa María, Lic. Karen Kateriny Medina Gamonal, Mg. Luis Flores Morillas, Lic. Mary Montenegro Medina, Mg. Gloria Juárez Farroñán, Lic. Kelly Medina Burga, Mg. Darío Hernández Quiroz, Mg. Judith Ascencio González. Asimismo, se agradece a la Comunidad Ser Maestro, la Universidad Santo Toribio de Mogrovejo, la Gerencia Regional de Educación de Lambayeque, el Colegio Militar Elías Aguirre. 


\section{Referencias}

Alonso, L. y Blázquez, F. (2016). El docente de educación virtual. Guía básica: Incluye orientaciones y ejemplos del uso educativo de Moodle (Vol. 33). Narcea Ediciones.

Alves, R., Lopes, T. y Precioso, J. Teachers' Well-Being in Times of Covid-19 Pandemic: Factors That Explain Professional Well-Being. IJERI: International journal of Educational Research and Innovation, (15), 203-217.

Arias, C. (2006) Enfoques teóricos sobre la, percepción que tienen las personas. Horizonte Pedagógica, 8(1), 9-22.

Castilla, C. A. A. (2006). Enfoques teóricos sobre la percepción que tienen las personas. Horizontes pedagógicos, 8(1), p. 1.

Banco Mundial (2018). Crisis de la educación: Informe de Desarrollo Mundial, Aprender para hacer realidad la promesa. Washington DC: Banco Mundial. https://openknowledge.worldbank.org/bitstream/ handle $/ 10986 / 28340 / 211096$ ov SP.PDF? sequence $=64$ \&is Allowed $=y$

Bautista, G., Borges, F. y Forés, A. (2006). Didáctica universitaria en entornos virtuales de enseñanza-aprendizaje (Vol. 13). Narcea Ediciones.

Cabrera, M. (5 de junio de 2020). COVID-19 y formación virtual: Cómo adoptar competencias digitales en cuestión de días. The Conversation. https:// theconversation.com/covid-19-y-formacion-virtual-como-adoptar-competencias-digitales-en-cuestion-de-dias-139698

Comisión Económica para América Latina y el Caribe/Fondo de las Naciones Unidas para la Infancia [Cepal/Unicef] (2020). La educación en tiempos de la pandemia de COVID 19. Desafíos, 23.

Cervera, M. G., Martínez, J. G., y Mon, F. M. E. (2016). Competencia digital y competencia digital docente: una panorámica sobre el estado de la cuestión. Revista Interuniversitaria de Investigación en Tecnología Educativa. https://doi.org/10.6018/riite2016/257631

Dans, E. (13 de abril de 2020). The Coronavirus Pandemic Has Unleashed A Revolution in Education: From Now On, Blended Learning Will Be The Benchmark. Revista Forbes. https://www.forbes.com/sites/enriquedans/2020/04/13/ the-coronavirus-pandemic-hasunleashed-a-revolution-in-education-fromnow-on-blended-learning-will-be-thebenchmark/\#44ecfeb6536f

Esteve Mon, F. M. (2015). La competencia digital docente: análisis de la autopercepción y evaluación del desempeño de los estudiantes universitarios de educación por medio de un entorno $3 D$ [Tesis de doctorado, Universitat Rovira i Virgili].

Failache, E., Katzkowicz, N., y Machado, A. (2020). La educación en tiempos de pandemia y el día después: El Caso de Uruguay. Revista Internacional de Educación para la Justicia Social, 9(3).

Ferrari, A. (2013). DIGCOMP: A Framework for Developing and Understanding Digital Competence in Europe. Institute for Prospective Technological

126 I Studies (IPTS), European Commission. 
Gehrig, R., y Muñoz Sánchez, P. (2015). Educación, Identidad y Derechos como estrategias de desarrollo de los Pueblos Indígenas. II Decenio de los Pueblos Indigenas 2005-2015. Editorial ECAM.

Gisbert, M., González, J. y Esteve, F. (2016). Competencia digital y competencia digital docente: una panorámica sobre el estado de la cuestión. RIITE. Revista Interuniversitaria de Investigación en Tecnología Educativa, 0, 74-83. Doi: http://dx.doi.org/10.6018/riite/2016/257631

Guterres, A. (4 de agosto de 2020). El impacto del COVID-19 en la educación podría desperdiciar un gran potencial humano y revertir décadas de progreso. UN News. https://news.un.org/es/story/2020/08/1478302

Hernández, L., Acevedo, J., Martínez, C. y Cruz, B. (2014). El uso de las TIC en el aula: un análisis en términos de efectividad y eficacia. Congreso Iberoamericano de Ciencia, Tecnología, Innovación y Educación. Recuperado de https://www.oei.es/historico/congreso2014/memoriactei/523.pdfINTEF

Hernández, R., Fernández, C. y Baptista, L. (2014). Metodología de la investigación. McGraw Hill.

Hinostroza, E. (2017). TIC, educación y desarrollo social en América Latina y el Caribe. Unesco.

Instituto Nacional de Tecnologías Educativas y de Formación del Profesorado [INTEF] (2017). Marco Común de Competencia Digital Docente. [Documento Oficial]. https://www.slideshare.net/educacionlab/marco-comn-decompetencia-digital-docente-2017

Instituto Peruano de Economía - El Comercio (24 de agosto 2020). Educación en los tiempos del covid-19. Instituto Peruano de Economía. https://www. ipe.org.pe/portal/educacion-en-los-tiempos-del-covid-19-aprendo-en-casa/.

Iordache, C., Mariën, I. y Baelden, D. (2017). Developing Digital Skills and Competences: A QuickScan Analysis of 13 Digital Literacy Models. Italian Journal of Sociology of Education, 9(1), 6-30. doi: https://doi.org/10.14658/ pupj-ijse-2017-1-2

Krumsvik, R. J. (2011). Digital Competence in the Norwegian Teacher Education and Schools. Högre Utbildning, 1(1), 39-51.

Luna, J. C. S. y Larrea, A. J. C. (2020). Propuesta de intervención psicocomunicativa en un contexto de emergencia sanitaria de una institución educativa pública. PAIAN, 11(2), 1-9.

Marza, M., y Cruz, E. (2018). Gaming como instrumento educativo para una educación en competencias digitales desde los Academic Skills Centres. Revista General de Información y Documentación, 28(2), 489-506. http://dx.doi. org/10.5209/RGID.60805

Mesa de Concertación para la Lucha contra la Pobreza - Lambayeque (2020). Consulta nacional: Propuestas regionales para el pacto Perú: propuesta de la región Lambayeque [Archivo PDF]. https://www.mesadeconcertacion. org.pe/storage/documentos/2020-10-07/consulta-nacionalparapactoperuaportes-desde-lambayeque.pdf 
Ministerio de Educación [Minedu] (2016). Currículo Nacional de la Educación Básica. Minedu.

Minedu, Currículo Nacional 2019). Programa Curricular de Educación secundaria. Minedu.

Ministerio de Educación [Minedu] (2020). Marco de Buen Desempeño Docente: Para mejorar tu práctica como maestro y guiar el aprendizaje de tus estudiantes [Documento oficial]. Minedu. http://www.minedu.gob.pe/pdf/ed/ marcode-buen-desempeno-docente.pdf

Moreno, A. G., Perez, J. J. C., Duque, S. A. O. y Gómez, J. J. O. (2021). Tecnoestrés laboral derivado de la virtualidad obligatoria por prevención del covid-19 en docentes universitarios de Medellín (COLOMBIA). Trabalho (En) Cena, e021003-e021003.

OECD (2019). Trends Shaping Education 2019. OECD Publishing. doi:https://dx.doi. org/10.1787/trends_edu-2019-en

Picón, G. A., de Caballero, K. G. y Paredes, N. (2020). Desempeño y formación docente en competencias digitales en clases no presenciales durante la pandemia COVID-19 [Archivo PDF].

Pimienta, D. (2007). Brecha digital, brecha social, brecha paradigmática. Funredes. http://www.funredes.org/mistica/castellano/ciberoteca/tematica/ brecha_paradigmatica.doc.

Pozos Pérez, K. V. y Tejada Fernández, J. (2018). Competencias digitales en docentes de educación superior: niveles de dominio y necesidades formativas. Revista Digital de Investigación en Docencia Universitaria, 12(2), 59-87.

Revelo Rosero, J. E., Revuelta Domínguez, F. I. y González-Pérez, A. (2018). Modelo de integración de la competencia digital del docente universitario para su desarrollo profesional en la enseñanza de la matemática - Universidad Tecnológica Equinoccial de Ecuador EDMETIC. Revista de Educación Mediática y TIC, 7(1), 196-224, doi: https://doi.org/10.21071/edmetic.v7i1.6910

Rugeles, P., Mora, B. y Metaute, P. (2015). El rol del estudiante en los ambientes educativos mediados por las TIC. Revista Lasallista de investigación, 12(2), 132-138. https://www.redalyc.org/pdf/695/69542291025.pdf

Ruiz, G. (2020). El derecho a la educación: Definiciones, normativas y políticas públicas revisadas. EUDEBA.

Salazar, P. (25 de mayo de 2020) Encuesta: La mitad de los profesores siente que no está logrando que los escolares aprendan lo esperado. Pontificia Universidad Católica de Chile - Noticias. https://www.uc.cl/noticias/encuestala-mitad-de-los-profesores-siente-que-no-esta-logrando-que-los-escolaresaprendan-lo-deseado/

Thomas, G. y Loxley, A. (2007). Deconstrucción de la educación especial y construcción de la inclusiva. Editorial La Muralla.

Vargas, A. L., Castro, J. A., y Alarcón, J. B. (2021). Digital Literacy and ICT In Secondary Education in Chile: Diagnosis in Times of Pandemic. Interciencia, 46(4), 148-155. 
Viner, R. M., Russell, S. J., Croker, H., Packer, J., Ward, J., Stansfield, C., Bonell, C. y Booy, T. (2020). School Closure and Management Practices During Coronavirus Outbreaks Including COVID19: A Rapid Systematic Review. The Lancet Child \& Adolescent Health, 4, 397-404. https://doi.org/10.1016/S23524642(20)30095-X 


\section{Apéndice}

Tabla 1

Docentes a quienes se aplicó la encuesta sobre percepción de competencias digitales docentes

\begin{tabular}{lcccccc}
\hline \multicolumn{1}{c}{ Provincia } & Varones & Mujeres & $\begin{array}{c}\text { Zona } \\
\text { urbana }\end{array}$ & $\begin{array}{c}\text { Zona } \\
\text { rural }\end{array}$ & $\begin{array}{c}\text { Gestión } \\
\text { pública }\end{array}$ & $\begin{array}{c}\text { Gestión } \\
\text { privada }\end{array}$ \\
\hline $\begin{array}{l}\text { Docentes de } \\
\text { Chiclayo }\end{array}$ & 16 & 20 & 28 & 08 & 30 & 06 \\
$\begin{array}{l}\text { Docentes de } \\
\text { Ferreñafe }\end{array}$ & 10 & 10 & 15 & 05 & 14 & 06 \\
$\begin{array}{l}\text { Docentes de } \\
\text { Lambayeque }\end{array}$ & 09 & 10 & 12 & 07 & 17 & 02 \\
\hline Total & 75 & 75 & & 75 & \\
\hline
\end{tabular}

Fuente: Encuesta de PCDD.

Tabla 2

Participantes a quiénes se aplicó entrevista a profundidad sobre percepción de competencias digitales docentes

\begin{tabular}{cccccc}
\hline Código & $\begin{array}{c}\text { Provincia } \\
\text { en la que } \\
\text { reside }\end{array}$ & Sexo & $\begin{array}{c}\text { Tiempo } \\
\text { de servicio }\end{array}$ & Edad & Formación \\
\hline E1 & Ferreñafe & Femenino & 30 & 53 & $\begin{array}{c}\text { Doctor en } \\
\text { Educación }\end{array}$ \\
E2 & Chiclayo & Masculino & 35 & 57 & $\begin{array}{l}\text { Doctor en } \\
\text { Educación }\end{array}$ \\
E3 & Lambayeque & Femenino & 32 & 54 & $\begin{array}{c}\text { Doctor en } \\
\text { Educación }\end{array}$ \\
\hline
\end{tabular}

Fuente: Entrevistas. 
Tabla 3

Participantes a quiénes se aplicó la entrevista a profundidad sobre percepción de competencias digitales docentes

\begin{tabular}{ccccc}
\hline Código & Característica & Sexo & Zona & Gestión \\
\hline Ia1 & Directivo & Femenino & Rural & Público \\
Ia2 & Directivo & Masculino & Urbana & Público \\
Ia3 & Directivo & Masculino & Urbana & Público \\
Ia4 & Docente & Femenino & Urbana & Público \\
Ia5 & Docente & Femenino & Rural & Público \\
Ia6 & Docente & Masculino & Urbana & Particular \\
Ia7 & Docente & Femenino & Rural & Público \\
Ia8 & Docente & Femenino & Urbana & Particular \\
Ib1 & Estudiante & Masculino & Urbana & Público \\
Ib2 & Estudiante & Femenino & Urbana & Público \\
Ib3 & Estudiante & Femenino & Urbana & Público \\
Ib4 & Estudiante & Femenino & Urbana & Público \\
Ib5 & Estudiante & Femenino & Urbana & Público \\
Ib6 & Estudiante & Femenino & Urbana & Público \\
\hline
\end{tabular}

Fuente: Grupo focales. 